\title{
Limited expression of TLR9 on T cells and its functional consequences in patients with nonalcoholic fatty liver disease
}

Nadia Soledad Alegre', Cecilia Claudia Garcia', Luis Ariel Billordo', Beatriz Ameigeiras², Daniel Poncino ${ }^{3}$, Javier Benavides ${ }^{4}$, Luis Colombato ${ }^{4}$, and Alejandra Claudia Cherñavsky ${ }^{1}$

${ }^{1}$ Instituto de Inmunología, Genética y Metabolismo, Universidad de Buenos Aires-Consejo Nacional de Investigaciones Científicas y Técnicas, Facultad de Farmacia y Bioquímica, Hospital de Clínicas “José de San Martín”, Buenos Aires; “Unidad de Gastroenterología, Hospital General de Agudos “JM Ramos Mejía”, Buenos Aires; ${ }^{3}$ Sección Hepatología, Servicio de Gastroenterología, Sanatorio Dr. “Julio Méndez", Buenos Aires; ${ }^{4}$ Sección Hepatología, Servicio de Gastroenterología, Hospital Británico de Buenos Aires, Buenos Aires, Argentina

\section{Graphical Abstract}
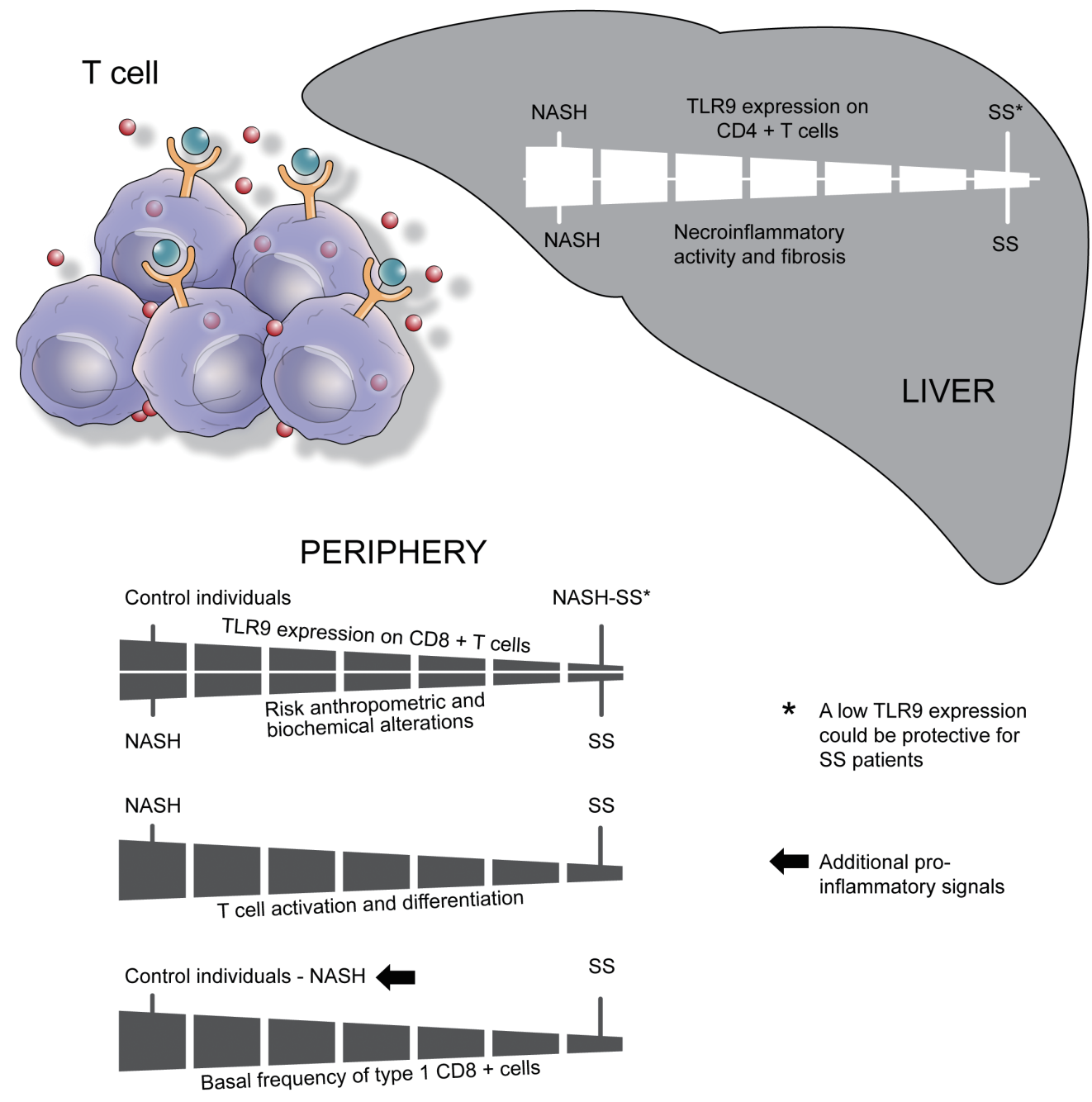

Copyright ( 92020 by Korean Association for the Study of the Liver

This is an Open Access article distributed under the terms of the Creative Commons Attribution Non-Commercial License (http://creativecommons.org/licenses/by-nc/3.0/) which permits unrestricted non-commercial use, distribution, and reproduction in any medium, provided the original work is properly cited. 
Background/Aims: Toll-like receptors (TLRs) modulate T cell responses in diverse diseases. Co-stimulation of T cell activation via TLR9 induces production of interferon gamma (IFN- $\gamma)$, priming of which is critical for differentiation of proinflammatory macrophages. These macrophages have a crucial role in nonalcoholic fatty liver disease (NAFLD). We aimed to evaluate the expression of TLR9 protein on T cells and the consequences of TLR9-mediated triggering of these cells in patients with NAFLD.

Methods: Our study included 34 patients with simple steatosis, 34 patients with nonalcoholic steatohepatitis, eight patients with NAFLD who met general diagnostic criteria but lacked histological diagnosis, and 51 control subjects. We used a synthetic TLR9 ligand to co-stimulate T cells. We measured TLR9 expression in liver and peripheral T cells and CD69 and IFN- $\gamma$ as phenotypic markers of T cell activation and differentiation by flow cytometry.

Results: TLR9 expression on liver and peripheral T cells was lowest in patients with simple steatosis and was positively associated with anthropometric, biochemical, and histopathological features of NAFLD. In vitro co-stimulation of T cells from patients with simple steatosis induced a limited number of IFN- $\gamma$-producing $C D 8^{+} T$ cells. At baseline, these patients showed a low frequency of circulating type $1 \mathrm{CD} 8^{+}$cells.

Conclusions: The positive associations between TLR9 and anthropometric, clinical, and histological features and the crucial role of IFN- $\gamma$-in NAFLD suggest that limited TLR9 expression and production of IFN- $\gamma$ play a protective role in patients with simple steatosis. (Clin Mol Hepatol 2020;26:216-226)

Keywords: Humans; Non-alcoholic fatty liver disease; CD8-positive T-lymphocytes; Toll-like receptor 9

\section{Study Highlights}

We demonstrated a limited expression of toll-like receptor 9 (TLR9) and interferon gamma (IFN- $-\gamma$ ) production by T cells in patients with simple steatosis (SS). The limited expression of TLR9 on T cells was directly associated with lower liver necroinflammatory activity and fibrosis, and lower anthropometric and biochemical alterations of nonalcoholic fatty liver disease. Besides, co-stimulation of T cell activation via TLR9 induced a limited number of IFN- $\gamma$-producing CD8 ${ }^{+} T$ cells in SS patients. Accordingly, these patients showed a low frequency of circulating type 1 CD $8^{+}$cells whereas additional pro-inflammatory signals may be responsible for the higher frequency observed at baseline in the context of steatohepatitis.

\section{INTRODUCTION}

Nonalcoholic fatty liver diseases (NAFLD) are a spectrum of liver diseases including simple steatosis (SS) and steatohepatitis as its more progressive form. ${ }^{1}$ The hallmark of nonalcoholic steatohepatitis (NASH) is the intrahepatic recruitment of inflammatory cells. All cells able to produce interferon gamma (IFN- $\gamma$ ) in inflammation can regulate the polarization of macrophages towards a pro-inflammatory phenotype. Compared with NASH, SS patients exhibit higher numbers of intrahepatic macrophages of anti-inflammatory nature. $^{2}$

Toll-like receptors (TLR) are a family of pattern recognition receptors able to activate the innate immune system. ${ }^{3}$ TLR9-mediated triggering of hepatic Kupffer cells is critically involved in NASH development ${ }^{4}$ firstly because NAFLD-associated small intestinal bacterial overgrowth 2,3 promotes the entry of TLR9 ligands of bacterial origin into the liver ${ }^{5}$ and secondly, due to the presence of microparticles of hepatocyte origin carrying mitochondrial DNA which also functions as TLR9 ligands. ${ }^{6}$

$T$ cell responses in diseases of diverse etiology are modulated

\author{
Abbreviations: \\ BMI, body mass index; CpG-ODN, cytidine-phosphate-guanosine \\ oligodeoxynucleotide; FITC, fluorescein isothiocyanate; IFN- $\gamma$, interferon \\ gamma; mAb, monoclonal antibody; MFI, mean fluorescence intensity; NAFLD, \\ nonalcoholic fatty liver diseases; NASH, nonalcoholic steatohepatitis; PBMC, \\ peripheral blood mononuclear cells; $\mathrm{PE}$, phycoerythrin; PerCP, Peridinin- \\ Chlorophyll-protein; SS, simple steatosis; TIR, toll-interleukin 1 receptor; TLR, toll- \\ like receptors
}

Editor: Sang Gyune Kim, Soonchunhyang University College of Medicine, Korea

\section{Corresponding author : Alejandra Claudia Cherñavsky}

Instituto de Inmunología, Genética y Metabolismo, Universidad de Buenos Aires-Consejo Nacional de Investigaciones Científicas y Técnicas, Facultad de Farmacia y Bioquímica, Hospital de Clínicas "José de San Martín", Avda. Córdoba 2351 (1120) CABA, Buenos Aires 1605, Argentina Tel: +54-011-5950 8756, Fax: +54-011-5950 8758

E-mail: alejandra.cher@yahoo.com.ar

https://orcid.org/0000-0001-8545-6944 
by TLRs expression, ${ }^{7-11}$ including TLR9. ${ }^{78} \mathrm{~T}$ cells co-stimulation via T cell receptor and TLR9 causes T cell activation and IFN- $\gamma$ production. ${ }^{12}$ We have previously identified the alteration of different T cell subsets in NASH patients ${ }^{13}$ and other authors confirmed a key role for T cells in NASH. ${ }^{14} \mathrm{~A}$ low intrahepatic expression of TLR9 mRNA was reported in SS. ${ }^{15}$ though it may probably come from the liver parenchyma than from T cells. So far, no study has evaluated TLR9 protein expression on T cells and the consequences of TLR9-mediated triggering in NAFLD. We hypothesized that T cells from patients with SS have a decreased expression of TLR9 and a limited production of IFN- $\gamma$.

\section{MATERIALS AND METHODS}

\section{Patients and general procedures}

Patients with NAFLD come from different centers of Buenos Aires whereas samples processing was entirely performed at the Clinical Hospital "José de San Martín". NAFLD patients group 1 comprised 68 individuals with biopsy-proven diagnosis of SS $(n=34)$ or NASH $(n=34)$. Its diagnosis was based on a daily alcohol consumption $<20 \mathrm{~g}$ (females) or $30 \mathrm{~g}$ (males), absence of other causes of liver disease and abdominal ultrasound. ${ }^{16}$ The histological diagnosis was based on the NAFLD Activity Score ${ }^{17}$ and fibrosis staging ${ }^{18}$ with score values $\geq 5$ as the cut-off for NASH. Liver biopsies and concomitant blood samples were used to evaluate TLR9 protein expression. NAFLD patients from group 2 were eight individuals who met the general criteria for NAFLD diagnosis but lacked histological diagnosis, hereafter called "NAFLD patients", used to evaluate TLR9 mRNA expression. Control group 1 included 11 metabolically healthy individuals who underwent antireflux surgery or cholecystectomy and did not have fatty liver which was confirmed by biopsy. Their biopsy samples were used to evaluate TLR9 protein expression. Control group 2 included 40 individuals recruited among the staff members of the Institute of Immunology, Genetics and Metabolism. All of the control individuals were given information of the study and those who intended to participate gave their written informed consent. Patients in the control groups did not received any medications within 6 months before the study, had low daily alcohol consumption, a body mass index (BMI) $<25 \mathrm{~kg} / \mathrm{m}^{2}$ and a waist circumference $<80 \mathrm{~cm}$ (females) or $94 \mathrm{~cm}$ (males). Their blood samples were used in different experiments as indicated. The BMI and waist circumference were evaluated according to the World Health Organization crite- ria. ${ }^{19}$ Homeostasis Assessment Model for insulin resistance was calculated as glucose $(\mathrm{mg} / \mathrm{dL}) \times$ insulin $(\mu \mathrm{U} / \mathrm{mL}) / 405$ and measures were performed using commercial kits. Plasma levels of total cholesterol and triglycerides, aspartate and alanine aminotransferases were determined in a Cobas C-501 autoanalyzer (Roche Diagnostics, Mannheim, Germany). As transaminases normal levels slightly vary depending on the individual laboratory's reference values, data were normalized before use. The experimental protocols and sample studies were approved by the local Ethics Committee (Clinic Hospital Ethics Committee, "Immunopathogenic factors that determine the progression of diseases associated with metabolic syndrome", March 6, 2017) which uses the internationally endorsed standards for the application of the Helsinki Declaration.

\section{Reagents}

We used Ficoll-Hypaque (Pharmacia Biotech, Uppsala, Sweden), TRIZOL® reagent and Roswell Park Memorial Institute 1640 medium (Life Technologies, Gaithersburg, MD, USA) and gentamicin, glutamine, ionomycin, fetal bovine serum, phorbol 12-myristate 13-acetate and brefeldin A (Sigma Chemical Co, Saint Louis, MO, USA). Phorbol 12-myristate 13-acetate was prepared in dimethyl sulfoxide at $25 \mu \mathrm{g} / \mathrm{mL}$. We used BDTM IMag anti-human CD3 magnetic particles, PermWash ${ }^{\mathrm{TM}}$, Cytofix/Cytoperm, anti-human CD3 monoclonal antibody (UCHT1) for cell stimulation, anti-TLR9phycoerythrin (PE) (eB72-1665) and anti-CD4-Peridinin-Chlorophyll-protein (PerCP) (SK3) from Becton-Dickinson (San Diego, CA, USA), anti-CD8-Fluorescein isothiocyanate (FITC) (UCHT-4), anti-CD69-PE (FN50) and anti-IFN- - -PE (B27) from ImmunoTools GmbH (Friesoythe, Germany). The synthetic cytidine-phosphateguanosine oligodeoxynucleotide (CpG-ODN) 2395 5'-tcgtcgttttcggcgcgcgccg-3' (Thermo Fisher Scientific, Waltham, MA, USA) used as TLR9 agonist was solubilized in sterile endotoxin-free water at $100 \mu \mathrm{M}$ and stored at 20X until use.

\section{Cell isolation}

Peripheral blood mononuclear cells were obtained using FicollHypaque gradients and resuspended in Roswell Park Memorial Institute 1640 medium 10\% fetal bovine serum, 2 mmol/L L-glutamine, and $50 \mu \mathrm{g} / \mathrm{mL}$ gentamicin. Twice-enriched fractions of $\mathrm{CD}^{+}$ $T$ cells were isolated from peripheral blood mononuclear cells (PBMC) by negative selection using magnetic particles. Recovered cells were at least $98 \% \mathrm{CD}^{+}$. We handled liver tissues at $4^{\circ} \mathrm{C}$ us- 
ing a hands-on homogenization method, washed liver cell suspensions twice in phosphate buffered saline and harvested cells by centrifugation.

\section{Quantitative polymerase chain reaction}

Total RNA was isolated from $\mathrm{CD}^{+}$cells using TRIZOL ${ }^{\circledR}$ reagent and reverse transcribed using oligo (dT) primers and SuperScript ${ }^{\mathrm{TM}}$ II First-Strand Synthesis System (Invitrogen, Life Technologies, Carlsbad, CA, USA). Forward and reverse primers: TLR9, 5'-GAAGGGACCTCGAGTGTGAAG-3' and 5'-GTAGGAAGGCAGGCAAGGTA-3'; glyceraldehyde 3-phosphate dehydrogenase, 5'-CGACCACTTTGTCAAGCTCA-3' and 5'-ACATGGCCTCCAAGGAGTAA-3'. Quantitative polymerase chain reaction was performed on a Stratagene Mx3005p RT-PCR Detection System (Agilent Technologies, La Jolla, CA, USA) using SYBR ${ }^{\circledR}$ Green PCR Master Mix (applied Biosystems, Life Technologies). Relative gene expression and fold change expression were calculated using the comparative Ct method. ${ }^{20}$

\section{Intracytoplasmic TLR9 protein measurement}

Suspension of $5 \times 10^{5}$ isolated peripheral blood mononuclear cells or liver cells was centrifuged at 2,000 rpm for 5 minutes and incubated with anti-CD4- PerCP and anti-CD8-FITC in darkness at $4^{\circ} \mathrm{C}$ for 20 minutes. Fixed and permeabilized cells were incubated with anti-TLR9-PE or the isotype control in darkness at $4^{\circ} \mathrm{C}$ for 40 minutes, washed twice in PermWash ${ }^{\mathrm{TM}} 1 \mathrm{X}$, resuspended in phosphate buffered saline $1 \mathrm{X}$ and assessed using flow cytometry. At least 100,000 events were acquired on a FACSCalibur flow cytometer (BD). The acquired data were analyzed using FlowJo software version 5.7.2 (Treestar, San Carlos, CA, USA). To analyze TLR9 expression, lymphocytes were gated on a forward and sidescatter plot and further gated based on CD4 or CD8 expression. TLR9 expression was expressed as a percent difference compared with isotype control using mean fluorescence intensity (MFI) values as follows: [(MFI of TLR9 / MFI of isotype control) - 1 / MFI of isotype control $\times 100]^{9}$

\section{CD69 measurement}

$1 \times 10^{6}$ isolated $\mathrm{CD}^{+}$cells were activated with soluble anti-CD3 (250 ng/mL) alone or in combination with $2 \mu \mathrm{M}$ CpG-ODN in 24well culture plates at $37^{\circ} \mathrm{C}$ for 24 hours. The cells were harvested by centrifugation, incubated with anti-CD4-PerCP, anti-CD8-FITC and anti-CD69-PE monoclonal antibodies in darkness at $4^{\circ} \mathrm{C}$,

Table 1. Demographic and baseline data of the study cohort

\begin{tabular}{|c|c|c|c|c|}
\hline \multirow{2}{*}{ Variable } & \multicolumn{3}{|c|}{ Group 1} & \multirow{2}{*}{$\begin{array}{c}\text { Group } 2 \\
\text { NAFLD }(n=8)\end{array}$} \\
\hline & SS $(n=34)$ & NASH $(n=34)$ & SS vs. NASH & \\
\hline Gender, M/F & $17 / 17$ & 15/19 & & $5 / 3$ \\
\hline Age (years) & $44(33-59)$ & $55(41-58)$ & NS & $39(25-51)$ \\
\hline $\operatorname{BMI}\left(\mathrm{kg} / \mathrm{m}^{2}\right)$ & $33.25(26.50-39.38)$ & $31.00(28.80-40.00)$ & NS & $31.23(28.96-42.60)$ \\
\hline Waist circumference (cm) & 110.00 (99.00-116.30) & $98.00(94.00-105.00)$ & $N S^{*}$ & $105.00(96.00-120.00)$ \\
\hline HOMA-IR & $4.50(2.30-8.20)$ & $4.95(3.10-7.85)$ & NS & $5.80(2.40-8.10)$ \\
\hline Fasting blood glucose (mg/dL) & $102.00(96.50-116.80)$ & 127.00 (98.00-137.00) & NS & $97.00(88.00-108.00)$ \\
\hline Insulin $(\mu \mathrm{U} / \mathrm{mL})$ & $16.00(10.60-30.50)$ & $21.00(9.12-26.50)$ & NS & $20.80(10.60-30.50)$ \\
\hline Total cholesterol (mg/dL) & $190.00(168.00-233.00)$ & $208.00(192.00-247.00)$ & NS & $185.00(163.00-224.00)$ \\
\hline Total triglycerides (mg/dL) & $153.00(88.50-218.50)$ & 165.00 (98.00-189.00) & NS & 153.00 (90.00-196.00) \\
\hline AST (UI/L) & $36.00(27.50-43.50)$ & $96.50(73.50-121.25)$ & 0.0003 & $75.00(28.00-121.00)$ \\
\hline $\operatorname{ALT}(\mathrm{UI} / \mathrm{L})$ & $47.50(32.75-58.25)$ & 137.00 (71.50-199.50) & 0.0028 & $77.50(41.25-165.80)$ \\
\hline
\end{tabular}

The table shows demographic and baseline data of NAFLD patients with biopsy-proven diagnosis of SS and NASH patients from group 1, and patients which met the general criteria for NAFLD but lacked the histological diagnosis from group 2. Results are summarized as median values (first-third quartiles). The Mann-Whitney $U$ test was used to compare continuous variables between SS and NASH patients. Reference values: BMI: $25.00-29.90 \mathrm{~kg} / \mathrm{m}^{2}$ (overweight), greater than $30.00 \mathrm{~kg} / \mathrm{m}^{2}$ (obesity). Waist circumference: $<84 \mathrm{~cm}$ (F) or $94 \mathrm{~cm}$ (M). HOMA-IR <2.5. Insulin: 2-20 $\mu \mathrm{U} / \mathrm{mL}$. Fasting blood glucose: $<100 \mathrm{mg} / \mathrm{dL}$. Total cholesterol: 150 to $199 \mathrm{mg} / \mathrm{d}$. Total triglycerides: <150 mg/dL. Aspartate transaminases: <48 UI/L. Alanine transaminases: <32 UI/L.

SS, simple steatosis; NASH, nonalcoholic steatohepatitis; NAFLD, nonalcoholic fatty liver diseases; M, male; F, female; BMI, body mass index; NS, no significant differences; HOMA-IR, homeostatic assessment model for insulin resistance; AST, aspartate transaminases; ALT, alanine transaminases.

${ }^{*}$ Comparisons were performed separately, between males or females with SS and NASH. 
washed twice and resuspended in phosphate buffered saline $1 \mathrm{X}$. A frequency index for $\mathrm{CD}_{6} 9^{+}$cells was calculated within $\mathrm{CD}^{+}$and $\mathrm{CD}^{+}$cell compartments as the ratio of frequencies observed after treatments with anti-CD3+CpG-ODN and anti-CD3 alone.

\section{Intracytoplasmic IFN- $\gamma$ staining}

To evaluate the frequency of IFN- $\gamma^{+}$cells at baseline, peripheral blood mononuclear cells were stimulated with $25 \mathrm{ng} / \mathrm{mL}$ phorbol 12-myristate 13-acetate and $1 \mathrm{mM}$ lonomycin in the presence of $1 \mathrm{mM}$ Brefeldin $\mathrm{A}$ at $37^{\circ} \mathrm{C}$ for 4 hours. After stimulation, cell sur-

Intrahepatic TLR9 expression

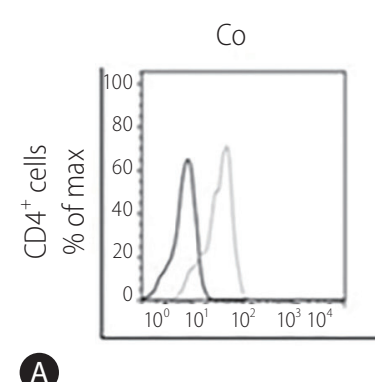

A
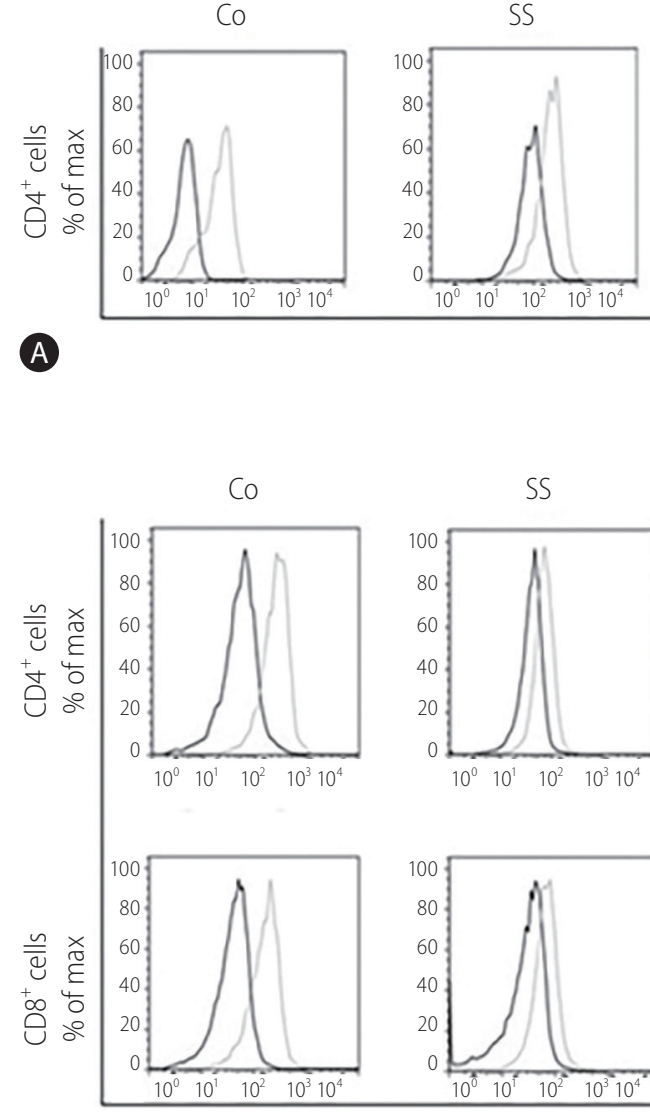

SS
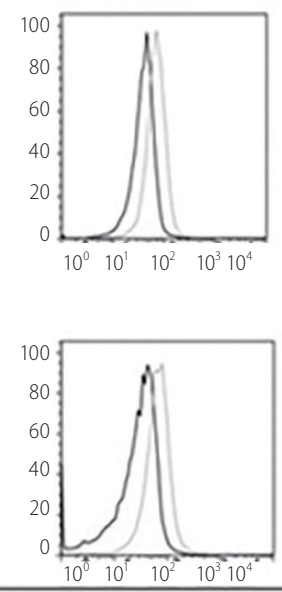

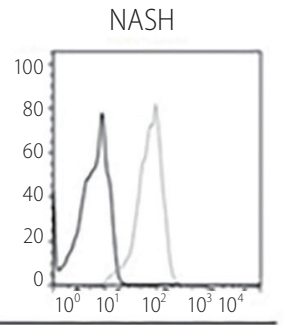

TLR9

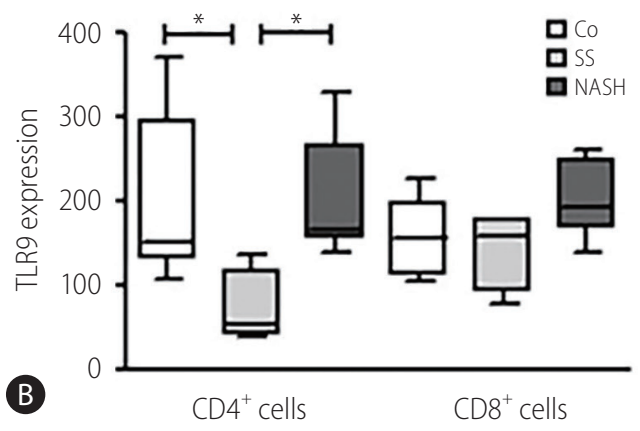

Peripheral TLR9 expression
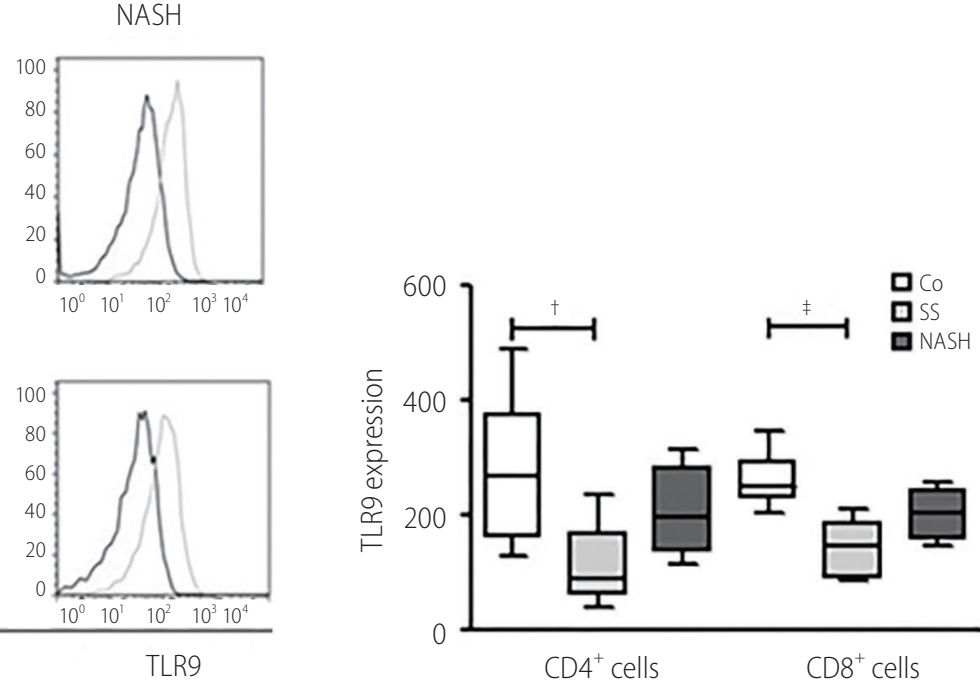

(D)

C $\longrightarrow$ Isotype control

Figure 1. Intrahepatic and peripheral TLR9 protein expression. Lymphocytes were gated on a forward and side-scatter plot, and the lymphoid region was further gated based on CD4 or CD8 expression (not shown). (A, B) Intrahepatic TLR9 expression. (A) Representative flow cytometric histograms showing TLR9 expression within a CD4 ${ }^{+}$cell compartment in a control subject, a patient with simple steatosis (SS), and a patient with nonalcoholic steatohepatitis (NASH). The ordinates and abscissas represent counts and fluorescence intensity, respectively. (B) The bar graph shows TLR9 expression in control subjects ( $n=11$, group 1), patients with SS ( $n=13$, group 1), and patients with NASH ( $n=14$, group 1). TLR9 expression was expressed as a percentage difference in mean fluorescence intensity (MFI) value compared with the isotype control as follows: [(MFI of TLR9 / MFI of isotype control-1 / MFI of isotype control $\times$ 100]. We used the Kruscal-Wallis with Dunn's multiple comparison test to compare data among the three groups. (C, D) Peripheral TLR9 expression. (C) Representative flow cytometric histograms showing TLR9 expression within intrahepatic CD4 ${ }^{+}$and CD8 ${ }^{+}$cell compartments in a control subject, a patient with SS, and a patient with NASH. (D) The bar graph shows TLR9 expression in control subjects and patients mentioned in (B). TLR9 expression was calculated as above. We used the Kruscal-Wallis with Dunn's multiple comparison test to compare data among the three groups. In (B) and (D), the lines in each box represent the median values, and the horizontal boundaries of the boxes represent the first and third quartiles. The vertical error bars show the minimum and maximum values. ${ }^{*} P<0.05 ;{ }^{\dagger} P=0.0219 ;{ }^{\ddagger} P=0.0022 \mathrm{vs}$. controls. 
face staining using anti-CD4-PerCP and anti-CD8-FITC monoclonal antibodies was performed as described above, and intracytoplasmic detection of IFN- $\gamma$ was done as described for TLR9. Lymphocytes were gated on a forward versus size scatter plot, the lymphoid region was further gated based on CD4 or CD8 expression, and IFN- $\gamma$ expression was measured in the selected populations. To evaluate the frequency of IFN- $\gamma^{+}$cells after co-stimulation, isolated PBMC were divided into $5 \times 10^{5}$ cells per well each containing $500 \mu \mathrm{L}$ of Roswell Park Memorial Institute medium and stimulated at $37^{\circ} \mathrm{C}$ for 18 hours in the presence of $1 \mathrm{mM}$ Brefeldin A with $250 \mathrm{ng} / \mathrm{mL}$ soluble anti-CD3 alone or anti-CD3+2 uM CpG-ODN in 24-well culture plates. A frequency index for IFN- $\gamma^{+}$cells was calculated as described for CD69.

\section{Statistical analysis}

We used GraphPad Prism software (GraphPad, San Diego, CA, USA). We performed the two-tailed Mann-Whitney $U$ test (two groups), the Kruskal-Wallis with Dunn's multiple comparison test
Table 3. TLR9 expression on circulating $C D 4^{+}$and $C D 8^{+}$cells and its relationship with biochemical and clinicopathological variables of NAFLD

\begin{tabular}{lcc}
\hline & CD4 $^{+}$ & CD8 $^{+}$ \\
\hline BMI* $^{*}$ & $0.6636(0.0260)$ & $0.8833(0.0016)$ \\
Waist circumference* $^{*}$ & NS & NS \\
\hline Fasting blood glucose* & NS & NS \\
\hline Total cholesterol* & NS & NS \\
\hline Total triglycerides* & NS & $0.9500(<0.0001)$ \\
AST* $^{*}$ & $0.7000(0.0037)$ & $0.6747(0.0081)$ \\
ALT* $^{*}$ & $0.6929(0.0042)$ & $0.7011(0.0052)$ \\
\hline
\end{tabular}

We used the Spearman's rank correlation coefficients $(r)$ to test the correlation between TLR9 expression and the anthropometric variables (BMI and waist circumpherence) or the plasma levels of selected metabolic variables, within peripheral $\mathrm{CD} 4^{+}$and $\mathrm{CD} 8^{+}$cell compartments. AST and ALT data were normalized according to the individual laboratory's reference values.

BMI, body mass index; NS, no significant differences; AST, aspartate aminotransferases; $A L T$, alanine aminotransferases.

${ }^{*}$ Results summarized as $r$ ( $P$-value).

Table 2. Relationships between TLR9 expression and necroinflammatory activity and fibrosis in NAFLD and comparison of TLR9 protein expression on intrahepatic T cells

\begin{tabular}{|c|c|c|c|}
\hline Cell type & Score $\leq 1$ & Score $\geq 2$ & $P$-value \\
\hline $\begin{array}{l}\text { A. Comparison between patients grouped according to } \\
\text { their histological scores of steatosis degree* }\end{array}$ & 14 & 13 & \\
\hline $\mathrm{CD}^{+}$ & $49.87(44.10-57.36)$ & $165.30(163.3-229.4)$ & 0.0042 \\
\hline $\mathrm{CD}^{+}$ & 179.30 (137.90-284.00) & $192.9(149.5-236.6)$ & NS \\
\hline $\begin{array}{l}\text { B. Comparison between patients grouped according to } \\
\text { their histological scores of ballooning degeneration* }\end{array}$ & 14 & 13 & \\
\hline $\mathrm{CD}^{+}$ & $53.6(41.2-138.2)$ & $165.4(162.6-248.1)$ & 0.0208 \\
\hline $\mathrm{CD}^{+}$ & $159.5(138.3-336.3)$ & $207.1(178.8-226.8)$ & NS \\
\hline $\begin{array}{l}\text { C. Comparison between patients grouped according to } \\
\text { their histological scores of lobular inflammation* }\end{array}$ & 19 & 8 & \\
\hline $\mathrm{CD}^{+}$ & $53.3(38.3-111.3)$ & $165.4(164.0-248.1)$ & 0.0227 \\
\hline $\mathrm{CD}^{+}$ & $179.3(139.7-284.0)$ & $207.1(178.8-226.8)$ & NS \\
\hline $\begin{array}{l}\text { D. Comparison between patients grouped according to } \\
\text { their fibrosis stage* }\end{array}$ & 16 & 11 & \\
\hline $\mathrm{CD}^{+}$ & $53.6(41.2-138.2)$ & $165.4(162.6-248.1)$ & 0.0381 \\
\hline $\mathrm{CD}^{+}$ & 159.5 (138.3-336.3) & 207.1 (178.8-246.4) & NS \\
\hline
\end{tabular}

The table shows the relationship between TLR9 expression on intrahepatic T cells and necroinflammatory activity (A-C) or fibrosis (D). NAFLD patients included in Fig. 1 ( $n=27$ ) were classified within two categories (as indicated in A-D), according to their histological scores of steatosis degree (A), ballooning degeneration (B), lobular inflammation (C), and fibrosis stage (D). Intrahepatic TLR9 protein expression was calculated as a percent difference compared with isotype control using MFI values as follows: [(MFI of TLR9 / MFI of isotype control - 1 / MFI of isotype control $\times 100]$. We used the Mann-Whitney test to compare TLR9 expression between two categories, $P<0.05$ was considered statistically significant.

NS, no significant differences; NAFLD, nonalcoholic fatty liver diseases; MFI, mean fluorescence intensity.

*Mean fluorescence intensity median values (first quartil-third quartil) are included in the table. 
(three or more groups), and Wilcoxon paired test (intragroup comparisons). Data were summarized using the median, first and third interquartiles and minimum and maximum values. We used the Spearman's rank correlation analysis to measure the statistical relations between TLR9 expression and continuous variables. $P<0.05$ was accepted as a significant value.

\section{RESULTS}

\section{Characteristics of patients with NAFLD and control subjects}

Anthropometric measurements, blood analysis and ultrasonography examination were performed at the beginning of the study (Table 1). All patients showed overweight or obesity and altered values of waist circumference and homeostasis assessment model for insulin resistance. We observed higher levels of aspartate and alanine aminotransferases in NASH than in SS patients from group 1. Anthropometric and biochemical values from control subjects fell into the reference ranges indicated (not shown). We described a direct relationship between plasma triglycerides concentration and fat content in NAFLD patients after comparing triglyceride levels between patients with steatosis scores " $\leq 1$ " and " $\geq 2$ " ( $P=0.0342$, not shown).

\section{Relationship between TLR9 expression, histological and clinicopathological features of NAFLD}

We assessed TLR9 protein expression in liver cell suspensions (Fig. 1A, B). TLR9 expression was lowest within the $C D 4^{+}$cell compartment in SS patients $(P<0.05$, vs. controls and vs. NASH patients) (Fig. 1B). TLR9 expression on $C D 8^{+} T$ cells was similar among groups. To get further insight into the biological relevance of these findings, we evaluated the relationship between TLR9 expression and NAFLD histological features (Table 2) after
Anti-CD3
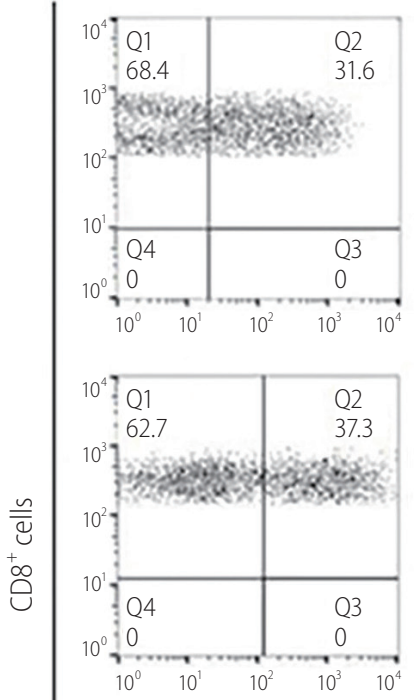

$\mathrm{NASH}$

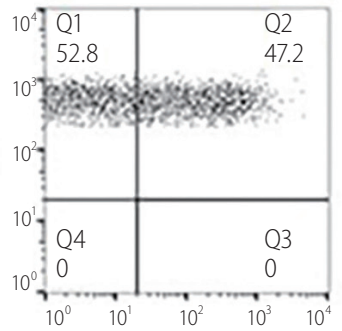

Anti-CD3 + CpG-ODN

Co

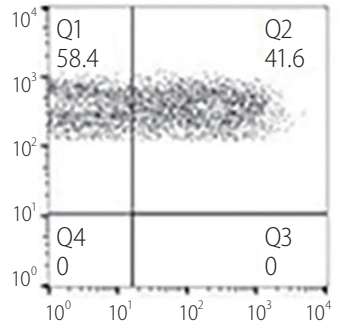

SS
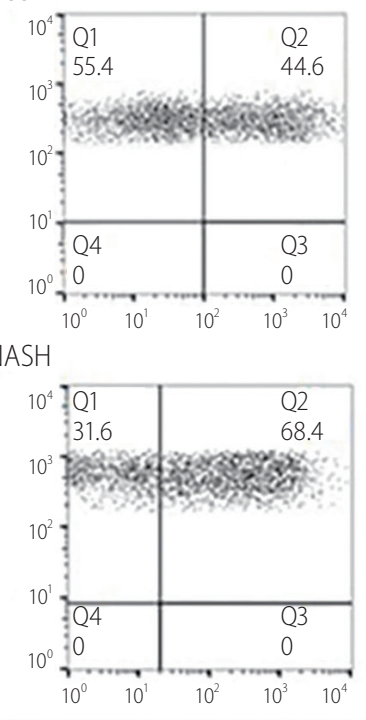

CD69

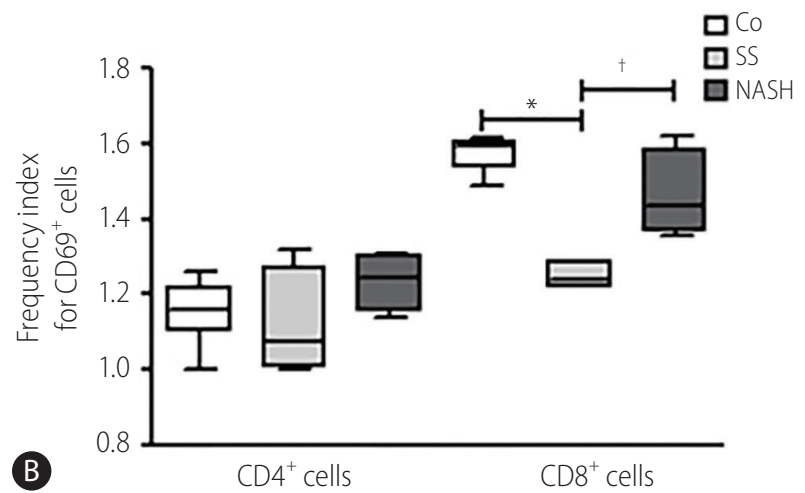

Figure 2. Synergistic interaction between T cell receptor and TLR9-mediated signaling in circulating cells. Isolated $\mathrm{CD}^{+}$cells from control subjects ( $n=6$, group 2), patients with simple steatosis (SS) ( $n=6$, group 1), and patients with nonalcoholic steatohepatitis (NASH) $(n=6$, group 1) were cultured in Roswell Park Memorial Institute 1640 medium, stimulated with anti-CD3 alone or with anti-CD3+CpG-ODN. After 24 hours of culture, surface staining of CD4, CD8, and CD69 antigens was performed. (A) Representative dot plots showing cells gated in the CD8 ${ }^{+}$region in a control subject, a patient with SS, and a patient with NASH. Dot plots show the percentage of $\mathrm{CD}^{+} 9^{+}$cells observed after treatment. (B) The frequency index for $\mathrm{CD}_{69}{ }^{+}$cells within $\mathrm{CD}^{+}$and $\mathrm{CD}^{+}$cell compartments was calculated as a ratio of frequencies observed after treatment with anti-CD3+CpG-ODN or anti-CD3 alone. The line in each box represents the median, the horizontal boundaries of the boxes represent the first and third quartiles, and the vertical error bars show the minimum and maximum values. We used the Kruscal-Wallis with Dunn's multiple comparison test to compare data among the three groups. ${ }^{*} P<0.005 ;{ }^{\dagger} P<0.0005$. 

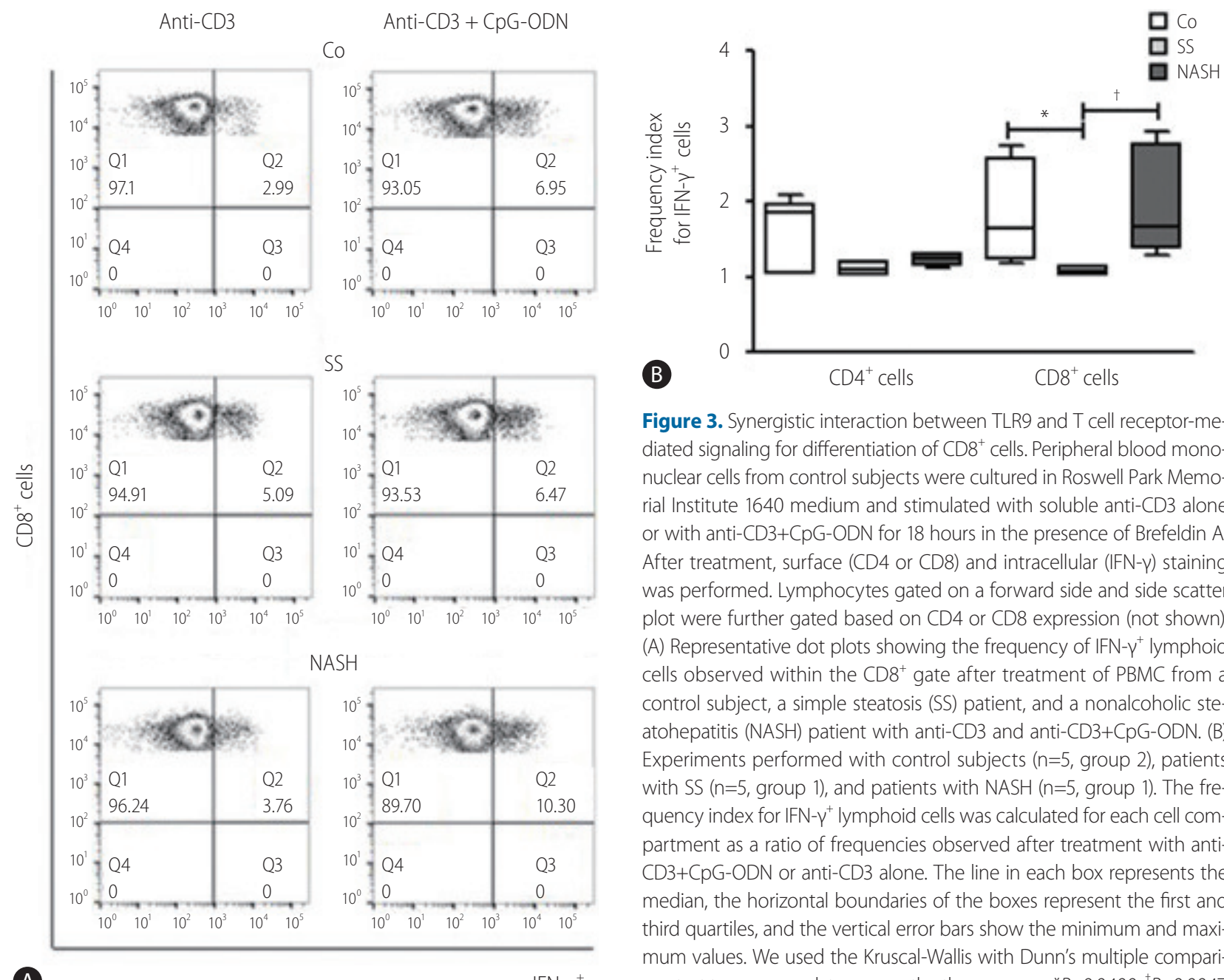

Figure 3. Synergistic interaction between TLR9 and T cell receptor-mediated signaling for differentiation of $\mathrm{CD}^{+}$cells. Peripheral blood mononuclear cells from control subjects were cultured in Roswell Park Memorial Institute 1640 medium and stimulated with soluble anti-CD3 alone or with anti-CD3+CpG-ODN for 18 hours in the presence of Brefeldin A. After treatment, surface (CD4 or CD8) and intracellular (IFN- - ) staining was performed. Lymphocytes gated on a forward side and side scatter plot were further gated based on CD4 or CD8 expression (not shown). (A) Representative dot plots showing the frequency of IFN- $\gamma^{+}$lymphoid cells observed within the $\mathrm{CD} 8^{+}$gate after treatment of PBMC from a control subject, a simple steatosis (SS) patient, and a nonalcoholic steatohepatitis (NASH) patient with anti-CD3 and anti-CD3+CpG-ODN. (B) Experiments performed with control subjects ( $n=5$, group 2$)$, patients with SS ( $n=5$, group 1), and patients with NASH ( $n=5$, group 1). The frequency index for IFN- $\gamma^{+}$lymphoid cells was calculated for each cell compartment as a ratio of frequencies observed after treatment with anti$\mathrm{CD} 3+\mathrm{CpG}-\mathrm{ODN}$ or anti-CD3 alone. The line in each box represents the median, the horizontal boundaries of the boxes represent the first and third quartiles, and the vertical error bars show the minimum and maximum values. We used the Kruscal-Wallis with Dunn's multiple comparison test to compare data among the three groups. ${ }^{*} P=0.0400 ;{ }^{\dagger} P=0.0047$.

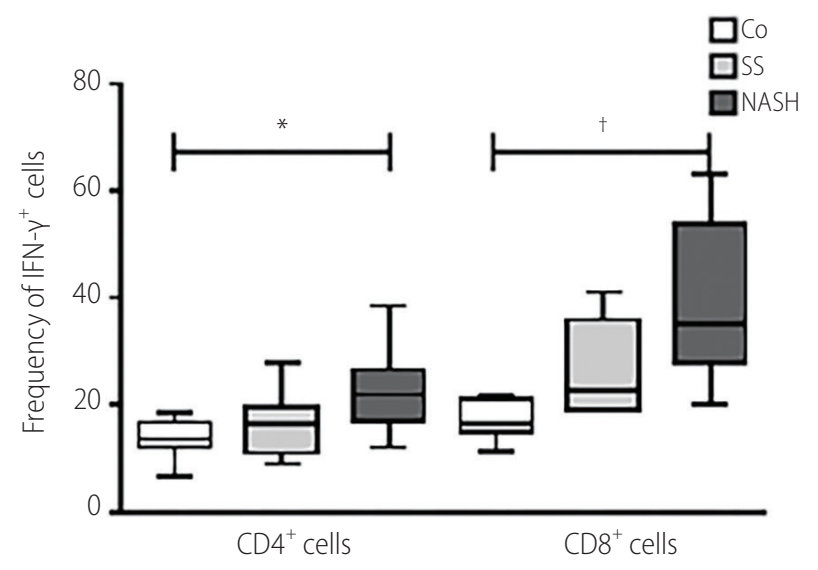

Figure 4. Frequency of IFN- $\gamma^{+}$circulating cells in control subjects and patients with NAFLD. Peripheral blood mononuclear cells from control individuals ( $n=11$, group 2), patients with simple steatosis (SS) ( $n=8$, group \#1), and patients with nonalcoholic steatohepatitis (NASH) ( $n=9$, group 1) were stimulated for 4 hours with the protein kinase $C$ stimulant phorbol 12-myristate 13-acetate and ionomycin in the presence of Brefeldin A before surface (CD4 or CD8) and intracellular (IFN- $\gamma$ ) staining. To analyze the frequency of IFN- $\gamma^{+}$ cells, lymphocytes gated on a forward side and side scatter plot were further gated based on CD4 or CD8 expression (not shown). The bar graph shows the percentage of IFN- $\gamma$-producing $\mathrm{CD}^{+}$and $\mathrm{CD} 8^{+}$cells. The line in each box represents the median, and the horizontal boundaries of the boxes represent the first and third quartiles. The vertical error bars show the minimum and maximum values, ${ }^{*} P<0.01 ;{ }^{\dagger} P=0.0005$. We used the KruscalWallis test to compare data among the three groups. 
stratification of NAFLD patients according to their histological scores within the categories indicated in Table 2. Our results showed a positive association between TLR9 expression in CD4 ${ }^{+}$ cells and liver damage. Then, we assessed TLR9 protein expression in peripheral blood mononuclear cells from the individuals previously evaluated (Fig. 1C, D). SS patients showed the lowest TLR9 expression within $C D 4^{+}(P=0.0219)$ and $C D 8^{+}(P=0.0022)$ cell compartments (Fig. 1D). A quantitative polymerase chain reaction-based analysis of TLR9 mRNA in $\mathrm{CD}^{+} \mathrm{T}$ cells confirmed a decreased expression of TLR9 mRNA in about $50 \%$ of patients (not shown). To assess the relationship between TLR9 expression at peripheral blood and clinic-pathological variables relevant to NAFLD, we performed a correlation analysis (Table 3). NAFLD patients with a lower alteration of BMI and aminotransferases activity exhibited a lower TLR9 expression within both cell compartments. We also found a positive association between plasma triglycerides concentration and TLR9 expression in peripheral $\mathrm{CD}^{+}$cells.

\section{Synergistic interaction between signals coming from T cell receptor and TLR9 in circulating T cells}

Given that a dysfunctionality of Kupffer cells in NASH ${ }^{21}$ might enhance the influx of TLR9 ligands into the systemic circulation and interact with T cells, we explored the consequences of TLR9mediated signaling of circulating $T$ cells. Because cell activation is a prerequisite for $\mathrm{T}$ cell differentiation, we assessed CD69 upregulation and IFN- $\gamma$ production as phenotypic markers of these processes. We employed isolated $\mathrm{CD}^{+}$cells for the assessment of cell activation to avoid the indirect effects of non-T cells, ${ }^{22}$ and the higher affinity of non-T cells for TLR9 ligands ${ }^{23}$ (Fig. 2). Regarding CD69 expression, the simultaneous addition of anti-CD3 and $\mathrm{CpG}-\mathrm{ODN}$ only provided co-stimulation to $\mathrm{CD} 8^{+}$cells (intragroup comparisons: anti-CD3+CpG-ODN vs. anti-CD3 alone, not shown). Upregulation of $C D 69$ was lowest in SS patients $(P<0.01$ vs. controls, $P<0.05$ vs. NASH patients). The addition of CpGODN did not affect CD69 expression (intragroup comparison: CpG-ODN treatment vs. medium, not shown). We employed peripheral blood mononuclear cells for cell differentiation assessment as a representative cellular platform that would allow the association of these results with the frequencies of IFN- $\gamma^{+}$producing cells at baseline (Fig. 3). Again, anti-CD3+CpG-ODN only provided co-stimulation for IFN- $\gamma$ production to $\mathrm{CD} 8^{+}$cells (intragroup comparisons: anti-CD3+CpG-ODN vs. anti-CD3 alone, not shown). Up-regulation of IFN- $\gamma^{+}$cells was lowest in SS pa- tients ( $P<0.05$ vs. controls, $P<0.01$ vs. NASH patients). As for CD69, the addition of CpG-ODN did not alter intracellular IFN- $\gamma$ production. Thus, our results demonstrated that T cells co-stimulation promoted the lowest activation and differentiation of circulating $\mathrm{CD} 8^{+}$cells in SS patients. The small size of our liver biopsies precluded its use for functional studies.

\section{Frequency of IFN- $\gamma^{+}$cells at baseline}

We measured the frequency of circulating IFN- $\gamma^{+} T$ cells (Fig. 4). The frequency of IFN- $\gamma^{+}$cells within $\mathrm{CD} 4^{+}(P<0.01)$ and $\mathrm{CD} 8^{+}$ $(P=0.0005)$ cell compartments was higher in NASH patients than control subjects. SS patients and control subjects showed similar frequencies of type $1 \mathrm{CD} 8^{+}$cells and Thelper 1 cells, respectively.

\section{DISCUSSION}

We found positive associations between TLR9 expression on intrahepatic $\mathrm{CD}^{+} \mathrm{T}$ cells, necroinflammation and liver fibrosis, and between TLR9 expression on peripheral $C D 4^{+}$and $C D 8^{+} T$ cells and clinic-pathological alterations of NAFLD such as BMI, plasma triglycerides concentration and aminotransferases activity. Relevant alterations of NAFLD in this study reflect liver disease or damage or predict the steatosis degree. ${ }^{24,25}$

The regulation of the inflammatory response in peripheral blood mononuclear cells is dependent on the metabolic status. ${ }^{26}$ Since metabolic alterations associated with NAFLD are similar among our patients, the decreased expression of TLR9 on T cells from SS patients may be independent from the metabolic context, particularly from insulin even though it down modulates the expression of TLRs on T cells. ${ }^{27}$

We showed an overall downregulation of TLR9 on T cells from SS patients affecting intrahepatic CD4 ${ }^{+} T$ cells as well as peripheral $\mathrm{CD}^{+}$and $C D 8^{+} T$ cells. It can be interpreted that this feature was likely a protection adaptation from hepatocellular injury whereas the observed unvarying expression in patients with NASH may be a failure of this regulatory mechanism. The cytoplasmic tails of all TLRs contain a toll-interleukin 1 receptor (TIR) domain. The TIR domain of TLR9 provides a binding site for an ubiquitin-protein ligase regulating its abundance and activity. ${ }^{28}$ Since ubiquitination of proteins mediates liver protection of patients with NAFLD, ${ }^{29}$ an increased ubiquitination of TLR9 might be involved in TLR9 downregulation on T cells from SS patients.

Our study confirms previous data concerning a synergism be- 
tween T cell receptors and TLR9 during the induction of IFN- ${ }^{12}$ Furthermore, it reveals a milder response in $\mathrm{CD}^{+}$cells from SS patients following its co-stimulation. Precise mechanistic insight exists to priming effects of IFN- $\gamma$ on macrophage activation towards an inflammatory phenotype. ${ }^{30}$ We can speculate that if the consequences of the co-stimulation were similar for intrahepatic and peripheral T cells, the decreased intrahepatic TLR9 expression observed in SS patients would promote a low number of type 1 CD8 ${ }^{+}$cells, favor the differentiation of anti-inflammatory Kupffer cells and protect against liver injury. In NASH, a failure of this regulatory mechanism would allow the progression of liver injury.

As compared with controls, NASH patients showed similar TLR9 expression and similar responses after TLR9 ligation of $T$ cells. However, NASH patients showed a higher frequency of IFN- $\gamma$ producing cells than SS patients and controls at baseline. In the context of NASH, the presence of strong pro-inflammatory adipokines and cytokines promoting type $1 \mathrm{CD}^{+}$cells differentiation ${ }^{31}$ supports these findings. The enrollment of patients in several centers from the specific area of Buenos Aires enhanced the possibility of generalizing our results to similar patients in similar settings. However, a limitation of our study was that a biopsy-proven diagnostic of NAFLD was unavailable from one of the participating centers. As we did not have an appropriate pilot study to estimate the sample size, we used information from previous studies in the same population, which allowed us to identify differences between groups provided that those differences genuinely existed in the population. 13,32

A decreased expression of TLR9 and a limited production of IFN- $\gamma$ by T cells from SS patients could play a protective role. T cells from NASH patients lack this mechanism and, furthermore, maybe influenced by strong pro-inflammatory signals of the microenvironment supporting the observed overproduction of IFN- $\gamma$. The impact of TLR9-mediated triggering of T cells within regulatory circuits potentially involved in NAFLD deserves further investigation.

\section{Authors' contribution}

NSA and ACCH designed the study; NSA, CCG and LAB performed the experiments and collected data; BA, DP and JB selected the patients, provided the samples and supervised clinical aspects; ACCH, LAB and NSA analyzed and interpreted the data; ACCH wrote the draft of the article; LC, NSA and ACCH made a critical revision and edited the article, acquired financial support, and made the final approval of the version to be published.

\section{Acknowledgments}

This research was supported by the University of Buenos Aires (grant numbers 100008BA and 00001BA) and the National Council for Scientific and Technological Investigation (grant number 0051).

\section{Conflicts of Interest}

The authors have no conflicts to disclose.

\section{REFERENCES}

1. Day CP. From fat to inflammation. Gastroenterology 2006;130:207210.

2. Wan J, Benkdane M, Teixeira-Clerc F, Bonnafous S, Louvet A, Lafdil F, et al. M2 Kupffer cells promote M1 Kupffer cell apoptosis: a protective mechanism against alcoholic and nonalcoholic fatty liver disease. Hepatology 2014;59:130-142.

3. Armant MA, Fenton MJ. Toll-like receptors: a family of pattern-recognition receptors in mammals. Genome Biol 2002;3:REVIEWS3011.1REVIEWS3011.6.

4. Miura K, Kodama Y, Inokuchi S, Schnabl B, Aoyama T, Ohnishi H, et al. Toll-like receptor 9 promotes steatohepatitis by induction of interleukin-1 $\beta$ in mice. Gastroenterology 2010;139:323-334.e7.

5. Rose II WA, Sakamoto K, Leifer CA. TLR9 is important for protection against intestinal damage and for intestinal repair. Sci Rep 2012:2:574.

6. Garcia-Martinez I, Santoro N, Chen Y, Hoque R, Ouyang X, Caprio $S$, et al. Hepatocyte mitochondrial DNA drives nonalcoholic steatohepatitis by activation of TLR9. J Clin Invest 2016;126:859-864.

7. Hammond T, Lee S, Watson MW, Flexman JP, Cheng W, Fernandez S, et al. Toll-like receptor (TLR) expression on CD4+ and CD8+ T-cells in patients chronically infected with hepatitis $C$ virus. Cell Immunol 2010;264:150-155.

8. Funderburg N, Luciano AA, Jiang W, Rodriguez B, Sieg SF, Lederman MM. Toll-like receptor ligands induce human $T$ cell activation and death, a model for HIV pathogenesis. PLoS One 2008;3:e1915.

9. Wong CK, Wong PT, Tam LS, Li EK, Chen DP, Lam CW. Activation profile of Toll-like receptors of peripheral blood lymphocytes in patients with systemic lupus erythematosus. Clin Exp Immunol 2010;159:11-22.

10. Riordan SM, Skinner N, Nagree A, McCallum H, Mclver CJ, Kurtovic $J$, et al. Peripheral blood mononuclear cell expression of toll-like receptors and relation to cytokine levels in cirrhosis. Hepatology 2003;37:1154-1164.

11. Babu S, Blauvelt CP, Kumaraswami V, Nutman TB. Cutting edge: diminished $T$ cell TLR expression and function modulates the immune 
response in human filarial infection. J Immunol 2006;176:38853889.

12. Kranzer K, Bauer M, Lipford GB, Heeg K, Wagner H, Lang R. CpGoligodeoxynucleotides enhance T-cell receptor-triggered interferon- $\gamma$ production and up-regulation of CD69 via induction of antigenpresenting cell-derived interferon type I and interleukin-12. Immunology 2000;99:170-178

13. Inzaugarat ME, Ferreyra Solari NE, Billordo LA, Abecasis R, Gadano $A C$, Cherñavsky AC. Altered phenotype and functionality of circulating immune cells characterize adult patients with nonalcoholic steatohepatitis. J Clin Immunol 2011;31:1120-1130.

14. Ilan Y, Shailubhai K, Sanyal A. Immunotherapy with oral administration of humanized anti-CD3 monoclonal antibody: a novel gutimmune system-based therapy for metaflammation and NASH. Clin Exp Immunol 2018;193:275-283.

15. Mridha AR, Haczeyni F, Yeh MM, Haigh WG, Ioannou GN, Barn V, et al. TLR9 is up-regulated in human and murine NASH: pivotal role in inflammatory recruitment and cell survival. Clin Sci (Lond) 2017;131:2145-2159.

16. Chalasani N, Younossi Z, Lavine JE, Diehl AM, Brunt EM, Cusi K, et al. The diagnosis and management of non-alcoholic fatty liver disease: practice Guideline by the American Association for the Study of Liver Diseases, American College of Gastroenterology, and the American Gastroenterological Association. Hepatology 2012;55:2005-2023.

17. Kleiner DE, Brunt EM, Van Natta M, Behling C, Contos MJ, Cummings $\mathrm{OW}$, et al. Design and validation of a histological scoring system for nonalcoholic fatty liver disease. Hepatology 2005;41:13131321.

18. Brunt EM, Janney CG, Di Bisceglie AM, Neuschwander-Tetri BA, Bacon BR. Nonalcoholic steatohepatitis: a proposal for grading and staging the histological lesions. Am J Gastroenterol 1999;94:24672474.

19. World Health Organization. Physical status: the use and interpretation of anthropometry. Report of a WHO Expert Committee. WHO Technical Report Series 854. Geneva: World Health Organization, 1995.

20. Schmittgen TD, Livak KJ. Analyzing real-time PCR data by the comparative C(T) method. Nat Protoc 2008;3:1101-1108.

21. Tonan T, Fujimoto K, Qayyum A, Morita Y, Nakashima O, Ono N, et al. CD14 expression and Kupffer cell dysfunction in non-alcoholic steatohepatitis: superparamagnetic iron oxide-magnetic resonance image and pathologic correlation. J Gastroenterol Hepatol 2012;27:789-796.

22. Prunk M, Perišić Nanut M, Sabotič J, Kos J. Cystatins, cysteine peptidase inhibitors, as regulators of immune cell cytotoxicity. Period Biol 2016;118:353-362.

23. Sharma RK, Sehgal S, Sachdeva N, Kumar R, Gupta A. Direct engagement of TLR9 ligand with T helper cells leads to cell proliferation \& up-regulation of cytokines. Immunol Invest 2019;48:79-95.

24. Angulo P, Keach JC, Batts KP, Lindor KD. Independent predictors of liver fibrosis in patients with nonalcoholic steatohepatitis. Hepatology 1999;30:1356-1362.

25. Fabbrini E, Magkos F, Mohammed BS, Pietka T, Abumrad NA, Patterson BW, et al. Intrahepatic fat, not visceral fat, is linked with metabolic complications of obesity. Proc Natl Acad Sci U S A 2009;106:15430-15435.

26. Isoni CA, Borges ÉA, Veloso CA, Mattos RT, Chaves MM, NogueiraMachado JA. CAMP activates the generation of reactive oxygen species and inhibits the secretion of IL- 6 in peripheral blood mononuclear cells from type 2 diabetic patients. Oxid Med Cell Longev 2009:2:3173-3121.

27. Ghanim H, Mohanty P, Deopurkar R, Sia CL, Korzeniewski K, Abuaysheh $\mathrm{S}$, et al. Acute modulation of toll-like receptors by insulin. Diabetes Care 2008;31:1827-1831.

28. Chuang TH, Ulevitch RJ. Triad3A, an E3 ubiquitin-protein ligase regulating Toll-like receptors. Nat Immunol 2004;5:495-502.

29. Zhu K, Tang Y, Xu X, Dang H, Tang LY, Wang X, et al. Non-proteolytic ubiquitin modification of PPARY by Smurf1 protects the liver from steatosis. PLoS Biol 2018;16:e3000091.

30. Wu C, Xue Y, Wang P, Lin L, Liu Q, Li N, et al. IFN- $\gamma$ primes macrophage activation by increasing phosphatase and tensin homolog via downregulation of miR-3473b. J Immunol 2014;193:3036-3044.

31. Stojsavljević S, Gomerčić Palčić M, Virović Jukić L, Smirčić Duvnjak $\mathrm{L}$, Duvnjak M. Adipokines and proinflammatory cytokines, the key mediators in the pathogenesis of nonalcoholic fatty liver disease. World J Gastroenterol 2014;20:18070-18091.

32. Inzaugarat ME, De Matteo E, Baz P, Lucero D, García CC, Gonzalez Ballerga $E$, et al. New evidence for the therapeutic potential of curcumin to treat nonalcoholic fatty liver disease in humans. PLoS One 2017;12:e0172900. 